\title{
Analysis of Superradiation Stability of Kerr-Newman Black Hole Using Curve integral
}

Wen-Xiang Chen ( $\nabla$ wxchen4277@qq.com )

GuangZhou University https://orcid.org/0000-0002-0560-8280

\section{Research Article}

Keywords: Cauchy integral, new variable y, superradiantly stable

Posted Date: November 17th, 2021

DOl: https://doi.org/10.21203/rs.3.rs-1072977/v2

License: (c) (i) This work is licensed under a Creative Commons Attribution 4.0 International License. Read Full License 


\title{
Analysis of Superradiation Stability of Kerr-Newman Black Hole Using Curve integral
}

\author{
Wen-Xiang Chen \\ Department of Astronomy, School of Physics and Materials Science, \\ GuangZhou University, Guangzhou 510006, China*
}

\begin{abstract}
In this article, a new variable $y$ is added here to expand the results of the above article. We use the properties of the Laurent series and the Cauchy integral. When y is greater than a certain limit, the effective potential of the equation does not have a pole, then there is no potential well outside the event horizon, when $\sqrt{2\left(a^{2}+Q^{2}\right)} / r_{+}^{2}<\omega<m \Omega_{H}+q \Phi_{H}$, so the Kerr-Newman black hole is superradiantly stable at that time.
\end{abstract}

\section{INTRODUCTION}

The No Hair Theorem of black holes first proposed in 1971 by Wheeler and proved by Stephen Hawking[1], Brandon Carter, etc, in 1973. In 1970s, the development of black hole thermodynamics applies the basic laws of thermodynamics to the theory of black hole in the field of general relativity, and strongly implies the profound and basic relationship among general relativity, thermodynamics and quantum theory. The stability of black holes is an major topic in black hole physics. Regge and Wheeler[2] have proved that the spherically symmetric Schwarzschild black hole is stable under perturbation. The great impact of superradiance makes the stability of rotating black holes more complicated. Superradiative effects occur in both classical and quantum scattering processes. When a boson wave hits a rotating black hole, chances are that rotating black holes are stable like Schwarzschild black holes, if certain conditions are satisfied[1-9]

$$
\omega<m \Omega_{H}+q \Phi_{H}, \Omega_{H}=\frac{a}{r_{+}^{2}+a^{2}}
$$

where $q$ and $m$ are the charge and azimuthal quantum number of the incoming wave, $\omega$ denotes the wave frequency, $\Omega_{H}$ is the angular velocity of black hole horizon and $\Phi_{H}$ is the electromagnetic potential of the black hole horizon. If the frequency range of the wave lies in the superradiance condition, the wave reflected by the event horizon will be amplified, which means the wave extracts rotational energy from the rotating black hole when the incident wave is scattered. According to the black hole bomb mechanism proposed by Press and Teukolsky[1-9], if a mirror is placed between the event horizon and the outer space of the black hole, the amplified wave will reflect back and forth between the mirror and the black hole and grow exponentially, which leads to the super-radiative instability of the black hole.

Later, it was discovered that the mass term of the scalar field and the Kaluza-Klein momentum of the ADS space-time boundary, the cosmological constant, and the infinitely high potential barrier outside the linear stretcher black hole can effectively act as a reflecting mirror (the role of a mirror can effectively prevent scattered waves from escaping to infinity. Far, it is provided by man, but also provided by nature itself). Under this mechanism, the superradiation wave will be reflected back and forth between the black hole horizon and the mirror and be amplified exponentially, thereby continuously extracting the rotation energy and Coulomb energy of the black hole, leading to the instability of the black hole. This property is called Black hole superradiation instability. In Kerr and R-N black holes, the superradiation qualitative theory of scalar field, electromagnetic field and gravitational wave was first given by Starobin-sky's classic paper. It is discussed there that due to the existence of the energy layer of the rotating black hole, its negative energy flow will enter the inner energy layer, and due to the conservation of energy, the outward scattered waves in the black hole will extract the energy of the black hole, and its amplitude will be increased. This provides a basis for the physical explanation of black hole superradiation.

Due to the existence of the superradiation mode, Press and Teukolsky proposed a black hole bomb mechanism. If there is a mirror between the black hole horizon and the space, the amplified wave can scatter back and forth and grow exponentially, which leads to superradiative instability under the black hole background. Specifically, a black hole bomb is the name of a physical effect. It is a physical phenomenon caused by a boson field amplified by superradiation scattering and hitting a rotating black hole. The additional condition that this phenomenon must meet is that the field must have a static mass that is not equal to zero. In this phenomenon, the scattered wave will be reflected back and forth between the mass interference term and the black hole, and will be amplified during each reflection. What we are interested in is the superradiation of the non-rotating charged RN black hole and the non-charged Kerr black hole.

*Electronic address: wxchen4277@qq.com 
For a rotating Kerr black hole, if the incoming scalar perturbation has a non-zero mass, then when the parameters of the Kerr black hole and the scalar field are in some parameter space, the mass term acts as a reflecting mirror and causes the superradiative instability of the Kerr black hole[1-10]. The literature also discusses the instability of the Kerr black hole affected by the mass vector field. Moreover, the literature also reported the superradiative instability of rotating black holes in curved space, such as Kerr-AdS black holes[1-9]. Despite so much research on the superradiation of rotating black holes, even Kerr black holes have not been fully discussed. Corresponding to the superradiation instability, many authors have studied the black hole stability of the superradiation mechanism. They adopted various methods to find the stability properties of the Kerr black hole under some scalar perturbations.

The superradiation scenario provides an interesting formation mechanism for BHsSH. Superradiance is very sensitive to scale matching. If the Boson's Compton wavelength $1 / \mu$ and the $\mathrm{BH}$ radius $\sim M$ approximately match, the instability is strongest: $M \mu \sim 1$. Away from this sweet spot, the time scale is unstable. The property increases exponentially with the $M \mu$ of $M \mu \gg 1[4]$, and increases with the large inverse power of $M \mu$ of $M \mu \ll 1$. Therefore, BHs becomes effectively hairy only in an island in the parameter space (on the astrophysical time scale), which is determined by the mass of the boson $\mu$. far enough from the resonance $M \mu \sim 1$, Kerr BHs Effective and stable to super radiation. Therefore, in this case, Kerr and hairy BHs may coexist in the universe. The latter belongs to an island in a narrow mass range near $\mu$ (and non-zero spin), and Kerr BHs make up the surrounding Ocean BH mass spectrometry.

The article[10] studies the superradiation stability of Kerr-Newman black holes and charged scalar disturbances. They treat black holes as background geometry and study the equations of motion for scalar perturbations. From the radial motion equation, we derive the effective potential experienced by the scalar disturbance. By a careful analysis of this effective potential, it is found that when the inner and outer horizons of Kerr-Newman black hole satisfy $\frac{r_{-}}{r_{+}} \leqslant \frac{1}{3}$ and the charge-to-mass ratios of scalar perturbation and black hole satisfy $\frac{q}{\mu} \frac{Q}{M}>1$, the Kerr-Newman black hole and scalar perturbation system is superradiantly stable.

Although there have been so much study on superradiance of rotating black holes, even Kerr black hole is not investigated thoroughly.Hod proved[12] that the Kerr black hole should be superradiantly stable under massive scalar perturbation when $\mu \geq \sqrt{2} m \Omega_{H}$, where $\mu$ is the mass.

In that article[43], a new variable y is added here to expand the results of the above article. When $\mu \geq \sqrt{2}\left(m \Omega_{H}+\right.$ $\left.q \Phi_{H}\right)$,so the Kerr-Newman black hole is superradiantly stable at that time.

In this article, a new variable $\mathrm{y}(\mu=y \omega)$ is added here to expand the results of the above article. We use the properties of curve integral. When $\mathrm{y}$ is greater than a certain limit, the effective potential of the equation does not have a pole, then there is no potential well outside the event horizon, when $\sqrt{2\left(a^{2}+Q^{2}\right)} / r_{+}^{2}<\omega<m \Omega_{H}+q \Phi_{H}$, so the KerrNewman black hole is superradiantly stable at that time.In Section 3, we outline the Kerr-Newman-black-hole-masivescalar system and the angular part of the equation of motion. We discussed some important asymptotic behaviors based on Schrodinger's effective potential and its derivatives, such as the effective potential of scalar perturbation in the radial equation and kn background in Section 4. Finally, we obtain the parameter space region of superradiation stability by analyzing the effective potential in detail in Section 5 .

\section{THE SUPERRADIATION EFFECT}

Superradiance in Flat Spacetime:We find the Klein-Gordon equation[36]

$$
\Phi_{; \mu}^{; \mu}=0
$$

where we defined $\Phi_{; \mu} \equiv\left(\partial_{\mu}-i e A_{\mu}\right) \Phi$ and $e$ is the charge of the scalar field. We get $A^{\mu}=\left\{A_{0}(x), 0\right\}$,and $e A_{0}(x)$ can be equal to $\mu$ (where $\mu$ is the mass).

$$
A_{0} \rightarrow\left\{\begin{array}{ll}
0 & \text { as } x \rightarrow-\infty \\
V & \text { as } x \rightarrow+\infty
\end{array} .\right.
$$

With $\Phi=e^{-i \omega t} f(x)$, which is determined by the ordinary differential equation

$$
\frac{d^{2} f}{d x^{2}}+\left(\omega-e A_{0}\right)^{2} f=0
$$

We see that particles coming from $-\infty$ and scattering off the potential with reflection and transmission amplitudes $\mathcal{R}$ and $\mathcal{T}$ respectively. With these boundary conditions, the solution to behaves asymptotically as

$$
\begin{gathered}
f_{\mathrm{i}}(x)=\mathcal{I} e^{i \omega x}+\mathcal{R} e^{-i \omega x}, x \rightarrow-\infty, \\
f_{\mathrm{n}}(x)=\mathcal{T} e^{i k x}, x \rightarrow+\infty
\end{gathered}
$$


where $k= \pm(\omega-e V)$.

To define the sign of $\omega$ and $k$ we must look at the wave's group velocity. We require $\partial \omega / \partial k>0$, so that they travel from the left to the right in the $x$-direction and we take $\omega>0$.

The reflection coefficient and transmission coefficient depend on the specific shape of the potential $A_{0}$. However one can easily show that the Wronskian

$$
W=\tilde{f}_{1} \frac{d \tilde{f}_{2}}{d x}-\tilde{f}_{2} \frac{d \tilde{f}_{1}}{d x}
$$

between two independent solutions, $\tilde{f}_{1}$ and $\tilde{f}_{2}$, of is conserved. From the equation on the other hand, if $f$ is a solution then its complex conjugate $f^{*}$ is another linearly independent solution. We find $|\mathcal{R}|^{2}=|\mathcal{I}|^{2}-\frac{\omega-e V}{\omega}|\mathcal{T}|^{2}$.Thus,for $0<\omega<e V$, it is possible to have superradiant amplification of the reflected current, i.e, $|\mathcal{R}|>|\mathcal{I}|$. There are other potentials that can be completely resolved, which can also show superradiation explicitly. )

We know that $\psi \sim \exp (-i \omega t+i m \phi)$, and the ordinary differential equation(We use tortoise coordinate $r_{*}, \frac{d r_{*}^{2}}{d r^{2}}=\frac{r^{2}}{\Delta}$

$$
\frac{d^{2} \psi}{d r_{*}^{2}}+V \psi=0
$$

There are other potentials that can be completely resolved, which can also show superradiation explicitly. We see that particles coming from $-\infty$ and scattering off the potential with reflection and transmission amplitudes $\mathcal{C}$ and $\mathcal{D}$ respectively. With these boundary conditions, the solution to behaves asymptotically as

$$
\psi=\left\{\begin{array}{l}
A e^{i \omega_{H} r_{*}}+B e^{-i \omega_{H} r_{*}}, r \rightarrow r_{+} \\
C e^{i \omega_{\infty} r_{*}}+D e^{-i \omega_{\infty} r_{*}}, r \rightarrow \infty
\end{array}\right.
$$

The reflection coefficient and transmission coefficient depend on the specific shape of the potential V.We show that the Wronskian

$$
W \equiv \psi \frac{d \bar{\psi}}{d r_{*}}-\bar{\psi} \frac{d \psi}{d r_{*}}
$$

$W\left(r \rightarrow r_{+}\right)=2 i \omega_{H}\left(|A|^{2}-|B|^{2}\right), W(r \rightarrow \infty)=2 i \omega_{\infty}\left(|C|^{2}-|D|^{2}\right)$ is conserved. We find $|C|^{2}-|D|^{2}=$ $\frac{\omega_{H}}{\omega_{\infty}}\left(|A|^{2}-|B|^{2}\right)$. Thus, for $\omega_{H} / \omega_{\infty}<0$, it is possible to have superradiant amplification of the reflected current, i.e, if $|A|=0,|\mathcal{C}|>|\mathcal{D}|$.

\section{THE SYSTEM OF KERR-NEWMAN BLACK HOLE}

The metric of the 4-dimensional Kerr-Newman black hole under Boyer-Lindquist coordinates $(t, r, \theta, \phi)$ is written as follow (with natural unit, $G=\hbar=c=1$ ) [11-24]

$$
\begin{aligned}
d s^{2} & =-\frac{\Delta}{\rho^{2}}\left(d t-a \sin ^{2} \theta d \phi\right)^{2}+\frac{\rho^{2}}{\Delta} d r^{2} \\
& +\rho^{2} d \theta^{2}+\frac{\sin ^{2} \theta}{\rho^{2}}\left[\left(r^{2}+a^{2}\right) d \phi-a d t\right]^{2},
\end{aligned}
$$

where

$$
\Delta \equiv r^{2}-2 M r+a^{2}+Q^{2} \quad, \quad \rho^{2} \equiv r^{2}+a^{2} \cos ^{2} \theta
$$

$a$ denotes the angular momentum per unit mass of certain Kerr-Newman black hole and $Q$, $M$ denote its charge and mass. The inner and outer horizons of the Kerr-Newman black hole can be expressed as

$$
r_{ \pm}=M \pm \sqrt{M^{2}-a^{2}-Q^{2}}
$$

and obviously

$$
r_{+}+r_{-}=2 M, \quad r_{+} r_{-}=a^{2}+Q^{2}
$$


We will study the physical and mathematical properties of the linearized large mass scalar field configuration (scalar cloud) with nontrivial coupling to the electromagnetic field of the Kerr-Newman black hole. The line element of space-time of spherically symmetric Kerr-Newman black hole can be expressed in the form of

$$
d s^{2}=-g(r) d t^{2}+\frac{1}{g(r)} d r^{2}+r^{2}\left(d \theta^{2}+\sin ^{2} \theta d \phi^{2}\right)
$$

where

$$
g(r)=1-\frac{2 M}{r}+\frac{a^{2}+Q^{2}}{r^{2}}
$$

The background electromagnetic potential is written as follow

$$
A_{\nu}=\left(-\frac{Q r}{\rho^{2}}, 0,0, \frac{a Q r \sin ^{2} \theta}{\rho^{2}}\right) .
$$

The following covariant Klein-Gordon equation

$$
\left(\nabla^{\nu}-i q A^{\nu}\right)\left(\nabla_{\nu}-i q A_{\nu}\right) \Phi=\mu^{2} \Phi
$$

where $\nabla^{\nu}$ represents the covariant derivative under the Kerr-Newman background. We adopt the method of separation of variables to solve the above equation, and it is decomposed as

$$
\Phi(t, r, \theta, \phi)=\sum_{l m} R_{l m}(r) S_{l m}(\theta) e^{i m \phi} e^{-i \omega t} .
$$

where $R_{l m}$ are the equations which satisfy the radial equation of motion (see Eq.(22) below). The angular function $S_{l m}$ denote the scalar spheroidal harmonics which satisfy the angular part of the equation of motion (see Eq.(20) below $). l(=0,1,2, \ldots)$ and $m$ are integers, $-l \leq m \leq l$ and $\omega$ denote the angular frequency of the scalar perturbation.

The angular part of the equation of motion is an ordinary differential equation and it can be expressed as follows,

$$
\begin{aligned}
& \frac{1}{\sin \theta} \frac{d}{d \theta}\left(\sin \theta \frac{d S_{l m}}{d \theta}\right) \\
& +\left[K_{l m}+\left(\mu^{2}-\omega^{2}\right) a^{2} \sin ^{2} \theta-\frac{m^{2}}{\sin ^{2} \theta}\right] S_{l m}=0,
\end{aligned}
$$

where $K_{l m}$ represent angular eigenvalues. The standard spheroidal differential equation above has been studied for a long time and of great significance in a great deal of physical problems. The spheroidal functions $S_{l m}$ are known as prolate (oblate) for $\left(\mu^{2}-\omega^{2}\right) a^{2}>0(<0)$, and only the prolate case is concerned in this article. We select the lower bound for this separation constant as follow, $[25-35]$

$$
K_{l m} \geq m^{2}-a^{2}\left(\mu^{2}-\omega^{2}\right) .
$$

The radial part of the Klein-Gordon equation contented by $R_{l m}$ is written as

$$
\Delta \frac{d}{d r}\left(\Delta \frac{d R_{l m}}{d r}\right)+U R_{l m}=0
$$

where

$$
\begin{aligned}
U= & {\left[\omega\left(a^{2}+r^{2}\right)-a m-q Q r\right]^{2} } \\
& +\Delta\left[2 a m \omega-K_{l m}-\mu^{2}\left(r^{2}+a^{2}\right)\right] .
\end{aligned}
$$

We get the following radial wave equation(another radial function $\psi=\mathrm{rR}$ )

$$
\frac{\mathrm{d}^{2} \psi}{\mathrm{dr}_{*}^{2}}+V \psi=0
$$

where

$$
V=\frac{U}{r^{4}}-\frac{2 \Delta}{r^{6}}\left(M r-\left(a^{2}+Q^{2}\right)\right) .
$$


V can change to $[17]$

$$
V(r)=\left(1-\frac{2 M}{r}+\frac{\left(a^{2}+Q^{2}\right)}{r^{2}}\right)\left[\mu^{2}+\frac{l(l+1)}{r^{2}}+\frac{2 M}{r^{3}}-\frac{2\left(a^{2}+Q^{2}\right)}{r^{4}}-\frac{\alpha\left(a^{2}+Q^{2}\right)}{r^{4}}\right]
$$

The study of the superradiant modes of KN black hole under the charged massive scalar perturbation require considering the asymptotic solutions of the radial equation near the horizon and at spatial infinity for appropriate boundary conditions. Here we adopt tortoise coordinate to analyse the boundary conditions for the radial function. The tortoise coordinate $r_{*}$ is defined by the following equation

$$
\frac{d r_{*}}{d r}=\frac{r^{2}+a^{2}}{\Delta} .
$$

The two boundary conditions we focused on are the purely ingoing wave next to the outer horizon and the exponentially decaying wave located at spatial infinity. Thus the asymptotic solutions of the radial wave function under the above boundaries are selected as follows

$$
R_{l m}(r) \sim \begin{cases}e^{-i\left(\omega-\omega_{c}\right) r_{*}}, & r^{*} \rightarrow-\infty\left(r \rightarrow r_{+}\right) \\ \frac{e^{-\sqrt{\mu^{2}-\omega^{2} r} r}}{r}, & r^{*} \rightarrow+\infty(r \rightarrow+\infty) .\end{cases}
$$

We can easily see that getting decaying modes at spatial infinity requires following bound state condition

$$
\omega^{2}<\mu^{2} .
$$

The critical frequency $\omega_{c}$ is defined as

$$
\omega_{c}=m \Omega_{H}+q \Phi_{H},
$$

where $\Omega_{H}$ is angular velocity of the outer horizon and $\Phi_{H}=\frac{Q r_{+}}{r_{+}^{2}+a^{2}}$ is the electric potential of whom.

\section{THE RADIAL EQUATION OF MOTION AND EFFECTIVE POTENTIAL}

A new radial wave function is defined as[11, 30-35]

$$
\psi_{l m} \equiv \Delta^{\frac{1}{2}} R_{l m} .
$$

in order to substitute the radial equation of motion (22) for a Schrodinger-like equation

$$
\frac{d^{2} \Psi_{l m}}{d r^{2}}+\left(\omega^{2}-V 1\right) \Psi_{l m}=0,
$$

where

$$
\omega^{2}-V 1=\frac{U+M^{2}-a^{2}-Q^{2}}{\Delta^{2}},
$$

in which $V$ denotes the effective potential.

Taking the superradiant condition (60), i.e. $\omega<\omega_{c}$, and bound state condition (28) into consideration, the KerrNewman black hole and charged massive scalar perturbation system are superradiantly stable when the trapping potential well outside the outer horizon of the Kerr-Newman black hole does not exist. As a result, the shape of the effective potential $V$ is analyzed next in order to inquiry into the nonexistence of a trapping well.

The asymptotic behaviors of the effective potential $V$ around the inner and outer horizons and at spatial infinity can be expressed as

$$
\begin{gathered}
V 1(r \rightarrow+\infty) \rightarrow \mu^{2}-\frac{2\left(2 M \omega^{2}-q Q \omega-M \mu^{2}\right)}{r}+\mathcal{O}\left(\frac{1}{r^{2}}\right), \\
V 1\left(r \rightarrow r_{+}\right) \rightarrow-\infty, \quad V 1\left(r \rightarrow r_{-}\right) \rightarrow-\infty .
\end{gathered}
$$

If a Kerr black hole satisfy the condition of $\mu=y \omega$, it will be superradiantly stable when $\mu<\sqrt{2} m \Omega_{H}$. In this article, we introduce the above condition into Kerr-Newman black holes. Therefore, the formula of the asymptotic behaviors is written as 


$$
\begin{gathered}
V 1(r \rightarrow+\infty) \rightarrow y^{2} \omega^{2}-\frac{2\left[M\left(2-y^{2}\right) \omega^{2}-q Q \omega\right]}{r}+\mathcal{O}\left(\frac{1}{r^{2}}\right), \\
V 1\left(r \rightarrow r_{+}\right) \rightarrow-\infty, \quad V 1\left(r \rightarrow r_{-}\right) \rightarrow-\infty
\end{gathered}
$$

It is concluded from the equations above that the effective potential approximates a constant at infinity in space, and the extreme between its inner and outer horizons cannot be less than one.The asymptotic behaviour of the derivative of the effective potential $V$ at spatial infinity can be expressed as

$$
V 1^{\prime}(r \rightarrow+\infty) \rightarrow \frac{2\left[M\left(2-y^{2}\right) \omega^{2}-q Q \omega\right]}{r^{2}}+\mathcal{O}\left(\frac{1}{r^{3}}\right)
$$

The derivative of the effective potential has to be negative in order to satisfy the no trapping well condition,

$$
2 M\left(2-y^{2}\right) \omega^{2}-2 Q q \omega<0
$$

\section{THE LIMIT $y$ OF THE INCIDENT PARTICLE UNDER THE SUPERRADIANCE OF KERR-NEWMAN BLACK HOLES}

Traditionally, universal gravitation is used to analogize superradiation phenomena, and superradiation is expected to realize the space-time rotation of toy models. A widely considered configuration is based on vortices, such as a drain bathtub; recently, the first experimental evidence of superradiation scattering has been obtained by using surface gravity waves in water to scatter such vortices. The situation, this is to understand the superradiation phenomenon from the Bose-Einstein condensation[42]. We have already said that the opposite arrow of this analogy is also very interesting. Here, we will use this point of view to reconsider the stability of the quantified vortex in the BEC, and study the special instabilities that occur in the non-uniform flow BEC similar to the hydrodynamic parallel shear flow.

Superradiance is very sensitive to scale matching. If the Boson's Compton wavelength $1 / \mu$ and the BH radius $\sim M$ approximately match, the instability is strongest: $M \mu \sim 1$. Away from this sweet spot, the time scale is unstable. The property increases exponentially with the $M \mu$ of $M \mu \gg 1[4]$, and increases with the large inverse power of $M \mu$ of $M \mu \ll 1$.

In the present section we shall derive a remarkably compact analytical formula for the discrete resonant spectrum $\left\{\alpha_{n}(\mu, l, M, a)\right\}_{n=0}^{n=\infty}$ which characterizes the composed Kerr-Newman black holes-linearized-massive-scalar-field configurations in the dimensionless large-mass regime[15]( $\alpha$ corresponds to the Laurent series to eliminate infinite transformations)

$$
M \mu \gg \max \{1, l\}
$$

As we will show clearly now, the Schrödinger-like equation determines the radial function behavior of the spacebounded non-minimum coupled mass scalar field configuration of Kerr-Newman black hole space-time, and is suitable for WKB analysis of large mass systems. In particular, Schrodinger-like standard second-order WKB analysis of the radial equation produces the well-known discrete quantization condition(Here we have used the integral relation $\int_{0}^{1} d x \sqrt{1 / x-1}=\pi / 2$. $)$, when $V(r \rightarrow+\infty), \mu=1 /\left(n+\frac{1}{2}\right)$,

$$
\int_{\left(y^{2}\right)_{t-}}^{\left(y^{2}\right)_{t+}} d\left(y^{2}\right) \sqrt{\omega^{2}-V 1(y ; M, a, l, \mu, \alpha 1)}=\left(n+\frac{1}{2}\right) \cdot \pi \mu / 2=\pi / 2 \quad ; \quad n=0,1,2, \ldots .
$$

The two integration boundaries $\left\{y_{t-}, y_{t+}\right\}$ of the WKB formula are the classical turning points $\left[\right.$ with $V\left(y_{t-}\right)=$ $V\left(y_{t+}\right)=0$ ] of the composed charged-black-hole-massive-field binding potential . The resonant parameter $n$ (with $n \in\{0,1,2, \ldots\})$ characterizes the infinitely large discrete resonant spectrum $\left\{\alpha_{n}(\mu, l, M, a)\right\}_{n=0}^{n=\infty}$ of the black-hole-field system. form

Using the relation between the radial coordinates $y$ and $r$, one can express the WKB resonance equation in the

$$
\int_{r_{t-}}^{r_{t+}} d r \frac{\sqrt{-V(r ; M, a, l, \mu, \alpha)}}{g(r)}=\left(n+\frac{1}{2}\right) \cdot \pi \quad ; \quad n=0,1,2, \ldots
$$


where the two polynomial relations

$$
1-\frac{2 M}{r_{t-}}+\frac{a^{2}+Q^{2}}{r_{t-}^{2}}=0
$$

and

$$
\frac{l(l+1)}{r_{t+}^{2}}+\frac{2 M}{r_{t+}^{3}}-\frac{2\left(a^{2}+Q^{2}\right)}{r_{t+}^{4}}-\frac{\alpha\left(a^{2}+Q^{2}\right)}{r_{t+}^{4}}=0
$$

determine the radial turning points $\left\{r_{t-}, r_{t+}\right\}$ of the composed black-hole-field binding potential.

We set

$$
x \equiv \frac{r-r_{+}}{r_{+}} \quad ; \quad \tau \equiv \frac{r_{+}-r_{-}}{r_{+}}
$$

in terms of which the composed black-hole-massive-field interaction term has the form of a binding potential well,

$$
V[x(r)]=-\tau\left(\frac{\alpha\left(a^{2}+Q^{2}\right)}{r_{+}^{4}}-\mu^{2}\right) \cdot x+\left[\frac{\alpha\left(a^{2}+Q^{2}\right)\left(5 r_{+}-6 r_{-}\right)}{r_{+}^{5}}-\mu^{2}\left(1-\frac{2 r_{-}}{r_{+}}\right)\right] \cdot x^{2}+O\left(x^{3}\right),
$$

in the near-horizon region

$$
x \ll \tau \text {. }
$$

From the near-horizon expression of the black-hole-field binding potential, one obtains the dimensionless expressions

$$
x_{t-}=0
$$

and

$$
x_{t+}=\tau \cdot \frac{\frac{\alpha\left(a^{2}+Q^{2}\right)}{r_{+}^{4}}-\mu^{2}}{\frac{\alpha\left(a^{2}+Q^{2}\right)\left(5 r_{+}-6 r_{-}\right)}{r_{+}^{5}}-\mu^{2}\left(1-\frac{2 r_{-}}{r_{+}}\right)}
$$

for the classical turning points of the WKB integral relation .

We find that our analysis is valid in the regime below $(\alpha 1$ corresponds to the transformation of $\mathrm{y}$ in Equation 38$)$

$$
\alpha \simeq \frac{\mu^{2} r_{+}^{4}}{\left(a^{2}+Q^{2}\right)}, \alpha 1 \simeq \sqrt{\frac{\mu^{2} r_{+}^{4}}{\left(a^{2}+Q^{2}\right)}}
$$

in which case the near-horizon binding potential and its outer turning point can be approximated by the remarkably compact expressions

$$
V(x)=-\tau\left[\left(\frac{\alpha\left(a^{2}+Q^{2}\right)}{r_{+}^{4}}-\mu^{2}\right) \cdot x-4 \mu^{2} \cdot x^{2}\right]+O\left(x^{3}\right)
$$

and

$$
x_{t+}=\frac{1}{4}\left(\frac{\alpha\left(a^{2}+Q^{2}\right)}{\mu^{2} r_{+}^{4}}-1\right) .
$$

In addition, one finds the near-horizon relation

$$
p(x)=\tau \cdot x+(1-2 \tau) \cdot x^{2}+O\left(x^{3}\right) .
$$

We know that

$$
\frac{1}{\sqrt{\tau}} \int_{0}^{x_{t+}} d x \sqrt{\frac{\frac{\alpha\left(a^{2}+Q^{2}\right)}{r_{+}^{2}}-\mu^{2} r_{+}^{2}}{x}-4 \mu^{2} r_{+}^{2}}=\left(n+\frac{1}{2}\right) \cdot \pi \quad ; \quad n=0,1,2, \ldots .
$$

Defining the dimensionless radial coordinate

$$
z \equiv \frac{x}{x_{t+}}
$$


one can express the WKB resonance equation in the mathematically compact form

$$
\frac{2 \mu r_{+} x_{t+}}{\sqrt{\tau}} \int_{0}^{1} d z \sqrt{\frac{1}{z}-1}=\left(n+\frac{1}{2}\right) \cdot \pi \quad ; \quad n=0,1,2, \ldots,
$$

which yields the relation

$$
\frac{\mu r_{+} x_{t+}}{\sqrt{\tau}}=n+\frac{1}{2} \quad ; \quad n=0,1,2, \ldots
$$

We know from the curve integral formula that there is a certain extreme value forming a loop

$$
1 / y^{2} \rightarrow \alpha
$$

y takes the interval from 0 to 1 at this time.

Interestingly, it has been demonstrated numerically in $[16,17]$ that the dimensionless physical parameter $\alpha$ diverges in the $y$ limit, where the physical parameter $y$ is defined by the dimensionless relation,for $y$ is greater than $\sqrt{2}$ at this time,

$$
y \equiv \alpha 1 / \sqrt{2} \text {. }
$$

Here the critical parameter y is given by the simple relation

$$
y / \mu \equiv \frac{r_{+}^{2}}{\sqrt{2\left(a^{2}+Q^{2}\right)}} .
$$

When

$$
\sqrt{2\left(a^{2}+Q^{2}\right)} / r_{+}^{2}<\omega<m \Omega_{H}+q \Phi_{H}, \Omega_{H}=\frac{a}{r_{+}^{2}+a^{2}}
$$

the Kerr-Newman black hole is superradiantly stable at that time.

\section{SUMMARY AND DISCUSSION}

In this article, we introduced $\mu=y \omega[38,39]$ into Kerr-Newman black holes, and discussed the superradiant stability of Kerr-Newman black holes. We adopt the variable separation method to divide the motion equation of the minimally coupled scalar perturbation in Kerr-Newman black hole into two forms: angular and radial.

The research[40] in 2012 shows that the small mass asymptotically non-flat Kerr-Newman-anti-de Sitter black holes produce superradiation instability under the scalar perturbation of charged mass. In 2016 [41], by analyzing the complex resonance spectrum of the charged mass scalar field in the near extremum Kerr-Newman black holes spacetime, the available dimensionless charge mass ratio is obtained $q / \mu$ to characterize the growth rate of super-radiative instability of scalar field, where $q$ is the charge of scalar field. [40] studied the small Kerr-Newman-anti-de Sitter black hole, and [41] studied the case without cosmological constant.The article[10] studies the superradiation stability of Kerr-Newman black holes and charged scalar disturbances. They treat black holes as background geometry and study the equations of motion for scalar perturbations.Hod proved[12] that the Kerr black hole should be superradiantly stable under massive scalar perturbation when $\mu \geq \sqrt{2} m \Omega_{H}$, where $\mu$ is the mass. In that article[43], when $\mu \geq$ $\sqrt{2}\left(m \Omega_{H}+q \Phi_{H}\right)$,so the Kerr-Newman black hole is superradiantly stable at that time.In this article, a new variable $\mathrm{y}$ is added here to expand the results of the above article. When $\sqrt{2\left(a^{2}+Q^{2}\right)} / r_{+}^{2}<\omega<m \Omega_{H}+q \Phi_{H}$, so the Kerr-Newman black hole is superradiantly stable at that time.

Acknowledgements:

I would like to thank Jing-Yi Zhang in GuangZhou University for generous help.This work is partially supported by National Natural Science Foundation of China(No. 11873025).

[1] Misner, Charles W.;Thorne, Kip S.;Wheeler, John Archibald.Gravitation. San Francisco:W. H. Freeman. $1973: 875-876$.

[2] T. Regge, J. A. Wheeler, Phys. Rev. 108, 1063(1957).

[3] W. Greiner, B. Mller, J. Rafelski, Quantum Electrodynamics of Strong Fields, Springer-Verlag, Berlin, 1985. 
[4] T. J. M. Zouros and D. M. Eardley, "Instabilities of massive scalar perturbations of a rotating black hole," Annals Phys., vol. 118, pp. 139-155, 1979.

[5] R. Penrose, Revista Del Nuovo Cimento, 1, 252 (1969).

[6] Ya. B. Zel'dovich, Pis'ma Zh. Eksp. Teor. Fiz. 14, 270 (1971) [JETP Lett. 14, 180 (1971)].

[7] W. H. Press and S. A. Teukolsky, Astrophys. J. 185, 649 (1973).

[8] A. V. Vilenkin, Phys. Lett. B 78, 301 (1978).

[9] Press W H, Teukolsky S A. Floating orbits, superradiant scattering and the black-hole bomb[J]. Nature, 1972, 238(5361): 211-212.

[10] Xu, Jun-Huai, et al. "Analytic study of superradiant stability of Kerr-Newman black holes under charged massive scalar perturbation." The European Physical Journal C 81.5 (2021): 1-8.

[11] Hod S. Stability of the extremal Reissner-Nordström black hole to charged scalar perturbations[J]. Physics Letters B, 2012, 713(4-5): 505-508.

[12] S. Hod, Phys. Lett. B 708, 320 (2012) [arXiv:1205.1872].

[13] R. P. Kerr, Phys. Rev. Lett. 11, 237 (1963).

[14] Degollado J C, Herdeiro C A R. Time evolution of superradiant instabilities for charged black holes in a cavity[J]. Physical Review D, 2014, 89(6): 063005.

[15] Hod, Shahar. "Reissner-Nordström black holes supporting nonminimally coupled massive scalar field configurations." Physical Review D 101.10 (2020): 104025.

[16] E. Berti, V. Cardoso and M. Casals, Phys. Rev. D 73, 024013 (2006) Erratum: [Phys. Rev. D 73, 109902 (2006)].

[17] C. A. R. Herdeiro, E. Radu, N. Sanchis-Gual, and J. A. Font, Phys. Rev. Lett. 121, 101102 (2018).

[18] M. Abramowitz and I. A. Stegun, Handbook of Mathematical Functions (Dover Publications, New York, 1970). 7

[19] J. M. Bardeen, W. H. Press, and S. A. Teukolsky, Astrophys. J. 178, 347 (1972).

[20] Shahar Hod. Physics Letters B, 2016, 758(C):181-185.

[21] Degollado J C, Herdeiro C A R, Rúnarsson H F. Rapid growth of superradiant instabilities for charged black holes in a cavity[J]. Physical Review D, 2013, 88(6): 063003.

[22] T. Damour, N. Deruelle and R. Ruffini, Lett. Nuovo Cimento 15, 257 (1976).

[23] T. M. Zouros and D. M. Eardley, Annals of physics 118, 139 (1979).

[24] S. Detweiler, Phys. Rev. D 22, 2323 (1980).

[25] H. Furuhashi and Y. Nambu, Prog. Theor. Phys. 112, 983 (2004).

[26] V. Cardoso and J. P. S. Lemos, Phys. Lett. B 621, 219 (2005).

[27] S. R. Dolan, Phys. Rev. D 76, 084001 (2007).

[28] S. Hod and O. Hod, Phys. Rev. D 81, Rapid communication 061502 (2010) [arXiv:0910.0734].

[29] S. Hod and O. Hod, e-print arXiv:0912.2761.

[30] S. Hod, Phys. Lett. B 739, 196 (2014) [arXiv:1411.2609].

[31] S. Hod, Phys. Lett. B 749, 167 (2015) [arXiv:1510.05649].

[32] S. Hod, Phys. Lett. B 751, 177 (2015).

[33] S. Hod, The Euro. Phys. Journal C 73, 2378 (2013) [arXiv:1311.5298].

[34] S. Hod, Class. and Quant. Grav. 32, 134002 (2015).

[35] C. A. R. Herdeiro and E. Radu, Phys. Rev. Lett. 112, 221101 (2014).

[36] Brito, Richard, Vitor Cardoso, and Paolo Pani. Superradiance. Springer International Publishing, 2020.

[37] Hod S. Stability of the extremal Reissner-Nordström black hole to charged scalar perturbations[J]. Physics Letters B, 2012, 713(4-5): 505-508.

[38] Chen, Wen-Xiang, and Zi-Yang Huang. "Superradiant stability of the kerr black hole." International Journal of Modern Physics D 29.01 (2020): 2050009.

[39] Valagiannopoulos, Constantinos. "Quantum fabry-perot resonator: Extreme angular selectivity in matter-wave tunneling." Physical Review Applied 12.5 (2019): 054042.

[40] Li, R. (2012). Superradiant instability of charged massive scalar field in Kerr-Newman-anti-de Sitter black hole. Physics Letters B, 714(2-5), 337-341.

[41] Hod, S. (2016). Analytic treatment of the system of a Kerr-Newman black hole and a charged massive scalar field. Physical Review D, 94(4), 044036.

[42] Luca Giacomelli. "Superradiant phenomena Lessons from and for Bose-Einstein condensates." https://iris.unitn.it. phd unitn luca giacomelli.

[43] Chen, Wen-Xiang, and Yi-Xiao Zhang. "The superradiant stability of Kerr-Newman black holes." arXiv preprint arXiv:2103.04239 (2021). 